\section{Insatisfação com o trabalho, aspectos psicossociais, satisfação pessoal e saúde mental de trabalhadores e trabalhadoras da saúde}

\author{
Job dissatisfaction, psychosocial aspects, \\ personal satisfaction, and mental health of male \\ and female health workers
}

\author{
Insatisfacción con el trabajo, aspectos \\ psicosociales, satisfacción personal y salud \\ mental de trabajadores y trabajadoras de salud
}

\author{
Camila Carvalho de Sousa 1 \\ Tânia Maria de Araújo 1 \\ Iracema Lua 2 \\ Mariana Rabelo Gomes 2 \\ Kátia Santana Freitas 1
}

\title{
Resumo
}

O objetivo foi avaliar a relação da insatisfação no trabalho e ocorrência de transtornos mentais comuns (TMC), considerando os aspectos psicossociais do trabalho e a satisfação pessoal (SAP). Estudo transversal, em amostra de 3.084 trabalhadores/as de saúde de cinco municípios baianos. Empregou-se análise de modelagem de equações estruturais, estratificada por gênero. A insatisfação com o trabalho foi a variável de exposição principal. Os TMC, variável desfecho, foram avaliados pelo Self-Reporting Questionaire (SRQ-20). Satisfação pessoal, avaliada por questões do World Health Organization Quality of Life (WHOQOL), e os aspectos psicossociais estressores (AE) e protetores $(A P)$, mensurados pelo Job Content Questionnaire (JCQ) e pela escala Effort-Reward Imbalance (ERI), foram tratadas como variáveis latentes. A insatisfação com o trabalho associou-se positivamente aos TMC, entre homens (0,160; IC95\%: 0,024; 0,295) e mulheres (0,198; IC95\%: 0,135; 0,260). Os AE associaram-se positivamente à insatisfação com o trabalho e aos TMC, enquanto os AP associaram-se negativamente a esses dois eventos, entre homens e mulheres. A insatisfação com o trabalho mediou o efeito dos aspectos psicossociais e da SAP na ocorrência de TMC. O modelo final apresentou bom ajuste nos grupos analisados. Elevada insatisfação, presença de aspectos psicossociais estressores, carência de aspectos protetores e insatisfação pessoal associaram-se aos TMC direta ou indiretamente. Os resultados reforçam a necessidade de ações voltadas à proteção da saúde mental no trabalho em saúde com o redesenho dos aspectos nocivos identificados e fortalecimento dos aspectos associados à menor ocorrência de TMC.

Estresse Ocupacional; Satisfação no Emprego; Transtornos Mentais

\author{
Correspondência \\ C. C. Sousa \\ Núcleo de Epidemiologia, Universidade Estadual de \\ Feira de Santana. \\ Av. Transnordestina $/$, Feira de Santana, $B A$ \\ 44036-900, Brasil. \\ camilacarvalho255@gmail.com \\ 1 Universidade Estadual de Feira de Santana, Feira de Santana, \\ Brasil. \\ 2 Instituto de Saúde Coletiva, Universidade Federal da Bahia, \\ Salvador, Brasil.
}




\section{Introdução}

O trabalho em saúde é uma atividade que envolve elevados níveis de estresse ocupacional. A exposição constante a fontes estressoras pode gerar insatisfação com o trabalho, com prejuízos à saúde mental dos/as trabalhadores/as 1,2,3. Em contrapartida, níveis elevados de satisfação com o trabalho podem contribuir para a diminuição da carga psíquica decorrente do estresse laboral, resultando em proteção à saúde mental do/a trabalhador/a 1.

A insatisfação com o trabalho contribui para a baixa autoestima, perda de interesse, irritabilidade, perda de energia vital e mau humor e comportamentos nocivos à saúde, que podem culminar em exaustão física e mental 4 . No caso do trabalho em saúde, no qual as atividades vinculam-se a intervenções na saúde das pessoas, a insatisfação pode influenciar o resultado do atendimento prestado aos usuários, com repercussão na qualidade e no alcance dos seus objetivos 5 .

A relação entre insatisfação com o trabalho e efeitos adversos na saúde mental dos/as trabalhadores/as tem sido observada na literatura em saúde e trabalho. Há evidências de que a insatisfação com a organização, a divisão e as condições nas quais se realiza o trabalho estão associadas ao adoecimento mental, de modo geral 2,3,6, e aos transtornos mentais comuns (TMC) 1,7, mais especificamente.

Os TMC caracterizam-se por sintomas como ansiedade, depressão, irritabilidade, dificuldade de concentração, esquecimento e fadiga 8 . A persistência dos sintomas compromete a qualidade de vida dos/as trabalhadores/as, contribui para elevar os índices de absenteísmo no trabalho, aumenta as demandas aos serviços de saúde e pode representar impactos econômicos significativos. Estimativas de TMC em diferentes contextos laborais destacam este evento como um importante problema de saúde pública 9,10,11.

Estudos têm revelado prevalências elevadas de TMC entre trabalhadores/as de saúde, com proporções que variaram de $16 \%$ a 46,9\%, em diferentes regiões do Brasil 11,12,13,14,15. Esse cenário evidencia a magnitude desse agravo, sendo necessário aprofundar o conhecimento sobre esse problema de saúde e seus determinantes.

O estresse ocupacional está relacionado à ocorrência de TMC 12 e com a insatisfação com o trabalho 1. Da mesma maneira, características relativas à satisfação pessoal (SAP) influenciam a satisfação laboral ${ }^{16}$. Contudo, não se sabe se há relação entre esses fatores, se atuam em conjunto ou isoladamente e se podem ou não modificar as situações desfavoráveis ou vantajosas à saúde mental em contextos laborais específicos.

O foco deste estudo é a análise da relação da insatisfação com o trabalho e a ocorrência de transtornos mentais comuns, levando-se em consideração os aspectos psicossociais do trabalho e a satisfação pessoal. Ou seja, além de focalizar a relação de insatisfação com o trabalho e TMC, pretende avaliar a contribuição de outros aspectos que a literatura aponta como associados à insatisfação e aos TMC. Pretende-se, assim, observar múltiplas relações que se estabelecem entre aspectos distintos do trabalho e da vida dos/as trabalhadores/as.

Neste estudo serão exploradas hipóteses sobre a relação entre insatisfação com o trabalho e saúde mental dos/as trabalhadores/as da saúde. A primeira hipótese diz respeito à associação entre a insatisfação com o trabalho e transtornos mentais comuns (assim, a insatisfação contribui diretamente para a ocorrência de TMC). A segunda assume que os aspectos psicossociais do trabalho (sejam eles estressores ou protetores) e a SAP podem associar-se positiva ou negativamente tanto à insatisfação quanto aos transtornos mentais, modificando a relação entre insatisfação com o trabalho e TMC. Desse modo, este estudo objetivou avaliar a relação entre insatisfação com o trabalho e a ocorrência dos transtornos mentais comuns, considerando os aspectos psicossociais do trabalho e a SAP.

Espera-se que os resultados obtidos contribuam para a consolidação de conhecimento na área da saúde do/a trabalhador/a, subsidiando a adoção de estratégias para elevar os níveis de satisfação com o trabalho, favorecendo a manutenção da saúde mental dos/as trabalhadores/as de saúde. 


\section{Metodologia}

Estudo transversal conduzido com 3.084 trabalhadores/as da saúde da atenção primária e de média complexidade de cinco municípios na Bahia, Brasil. É um projeto multicêntrico, envolvendo cinco instituições baianas de Ensino Superior. A coleta de dados foi realizada nos anos de 2011 a 2012.

A amostra foi definida em etapas sucessivas: (a) obteve-se a lista nominal dos/as trabalhadores/as em atividade nos serviços de saúde da atenção básica e da média complexidade; (b) estimou-se o tamanho amostral com base no total da população e em diferentes desfechos de saúde; (c) estratificou-se a amostra por três níveis: área geográfica, nível de assistência (atenção básica e média complexidade) e grupo ocupacional; (d) realizou-se o sorteio dos/as trabalhadores/as por meio de listagem de números aleatórios do Epi Info 6.04d (https://www.cdc.gov/epiinfo/index.html). Após o sorteio, os/as trabalhadores/as foram contatados/as nos próprios locais de trabalho, sendo realizadas até três visitas para que a entrevista fosse realizada. A coleta de dados utilizou questionário estruturado, previamente testado em estudo piloto.

Em cada município foi formada uma equipe de pesquisa sob a coordenação das pesquisadoras das universidades e serviços de saúde em cada local. A gestão do projeto incluiu a coordenação geral e as coordenações dos municípios.

A padronização metodológica foi estabelecida por um manual de procedimentos e condutas e oficinas de treinamento da equipe para a coleta dos dados. Nessa etapa, foram definidas as estratégias para a entrada nos locais de trabalho, sensibilização para a participação no estudo (campanha informativa e de divulgação da pesquisa) e abordagem do/a trabalhador/a (contato inicial e entrevista). A equipe foi composta por bolsistas de iniciação científica, discentes da pós-graduação e docentes das universidades envolvidas. Após a coleta, realizou-se dupla digitação dos questionários para avaliar possíveis inconsistências e garantir a qualidade do banco de dados.

No estudo multicêntrico, definiu-se como critérios de inclusão: estar em efetivo exercício profissional, aceitar participar da pesquisa e ter tempo mínimo de seis meses de trabalho na unidade. Os/ as trabalhadores/as não encontrados/as após três tentativas, que se recusaram a participar do estudo ou estavam de licença durante o período de coleta de dados foram substituídos/as, respeitando-se a área geográfica, o nível de complexidade do serviço, o grupo ocupacional e o sexo. Esses critérios de inclusão e exclusão foram adotados neste estudo.

A fim de verificar se o estudo multicêntrico tinha poder para avaliar a associação entre insatisfação com o trabalho e TMC, uma vez que a amostra não havia sido estimada com esta finalidade, o tamanho amostral foi recalculado. O cálculo foi realizado utilizando-se o software OpenEpi (http:// www.OpenEpi.com), versão 3.03a. Considerou-se a prevalência de TMC entre o grupo de expostos de $45 \%$ e entre o grupo de não expostos de $17 \%$ 1, nível de $95 \%$ de confiança e poder do estudo de 90\%, obteve-se o tamanho amostral de 160 trabalhadores/as. A amostra necessária foi acrescida de $20 \%$, considerando-se as possíveis perdas, totalizando 192 trabalhadores/as. Com a finalidade de aumentar o poder da pesquisa, foram incluídos todos/as os/as 3.084 trabalhadores/as participantes do estudo multicêntrico.

A insatisfação com o trabalho, variável de exposição principal, foi avaliada por meio da questão: Você está satisfeito/a com o seu trabalho? As opções de resposta variaram de 1 a 4: 1 = não estou satisfeito/a de forma nenhuma; 2 = não estou satisfeito/a; 3 = estou satisfeito/a; e 4 = estou muito satisfeito/a. Essa variável foi dicotomizada em satisfeito/a (valores 3 e 4) e insatisfeito/a (1 e 2).

Considerando que satisfação e insatisfação representam sentidos opostos de um mesmo fenômeno, é pertinente a utilização desse tipo de escala para avaliar a insatisfação, sendo um procedimento já empregado em outros estudos 1 nos quais verificou-se a adequação de medir a satisfação com o trabalho com uma única questão. Abordagens desse tipo apresentaram correlação positiva com as medidas realizadas com base em questionários multidimensionais ${ }^{17}$. Ademais, a utilização desse procedimento para estimar a insatisfação com o trabalho apresenta outras vantagens, tais como: pode conter maior validade e são melhores em capturar mudanças na satisfação com o trabalho ${ }^{18}$. Desse modo, ainda que a avaliação da insatisfação tenha sido feita por uma única questão, há sustentação na literatura que apoia o seu uso como um bom indicador de estado geral de insatisfação com o trabalho.

Os TMC, variável desfecho, foram avaliados pelo Self-Reporting Questionnaire (SRQ-20). Trata-se de um instrumento autoaplicável, composto de 20 questões mensuradas em escala dicotômica (sim/ 
não), que permite rastrear indivíduos com TMC, sendo recomendado, como ponto de corte, sete ou mais respostas positivas para mulheres e cinco ou mais respostas positivas para homens 19.

As variáveis relativas à SAP, aspectos psicossociais do trabalho estressores (AE) e aspectos psicossociais do trabalho protetores (AP) foram tratadas como variáveis latentes, mensuradas por meio de questões do World Health Organization Quality of Life (WHOQOL), das dimensões do Job Content Questionnaire (JCQ) e da escala Effort-Reward Imbalance (ERI), respectivamente. O termo variável latente diz respeito a um construto que não pode ser diretamente medido, mas pode ser mensurado ou representado por um conjunto de variáveis indicadoras 20. As variáveis latentes permitem acessar construtos difíceis de serem medidos, sendo uma alternativa para a análise de fatores subjetivos.

O JCQ avalia dimensões psicossociais do trabalho. Foram analisadas quatro dessas dimensões: demanda psicológica, avalia as exigências enfrentadas na execução das atividades laborais (ritmo de trabalho, volume de tarefas, tempo suficiente); controle sobre o próprio trabalho (incluindo o uso de habilidades e autonomia no processo decisório); apoio social proveniente da chefia e dos colegas de trabalho e demanda física, relativa ao esforço físico despendido na realização das tarefas 21 . Foi utilizada a versão recomendada de 49 itens, mensurados em escala do tipo Likert, variando de 1 a 4 ( $1=$ discordo fortemente; 2 = discordo; 3 = concordo e 4 = concordo fortemente). Obtidos os escores de cada escala procedeu-se à sua dicotomização com base na mediana (valores $\leq$ mediana = não exposto; valores $>$ mediana $=$ exposto), compondo as categorias: demanda baixa/alta, controle baixo/alto, demanda física baixa/alta e apoio social baixo/alto).

O modelo ERI avalia esforço e recompensa no trabalho. Esforço refere-se à energia despendida para a realização do trabalho, envolve gasto físico e psíquico; recompensa avalia retornos como os ganhos financeiros, percepção de reconhecimento e respeito no ambiente laboral, expectativas de promoção, compatibilidade do cargo ocupado com a formação e sentimento de justiça nas relações. O ERI tem uma dimensão adicional, comprometimento excessivo com o trabalho, que corresponde a um fator intrínseco, subjetivo, que se caracteriza pelo esforço excessivo, visando ao reconhecimento e à aprovação 22 . O modelo ERI é construído com base em um questionário autoaplicável de 23 itens, respondido em escala do tipo Likert ( 1 = discordo fortemente; 2 = discordo; 3 = concordo; 4 = concordo fortemente). A mediana foi usada como ponto de corte para a dicotomização das escalas propostas para a composição de esforço (baixo/alto), recompensa (baixa/alta), comprometimento excessivo (ausente/presente).

Para a mensuração das variáveis latentes, procedeu-se à avaliação conjunta das dimensões do ERI e do JCQ. A combinação desses modelos tem sido descrita na literatura como estratégia promissora à investigação de desfechos em saúde, tendo como pressuposto que considerar as diferentes exposições estabelecidas em cada um desses modelos permite superar as limitações dos modelos isolados 12,23,24,25. Neste estudo, abordou-se a junção das dimensões dos dois modelos para a composição das variáveis latentes. É uma metodologia inovadora, na qual espera-se que o conjunto dessas dimensões seja capaz de mensurar os aspectos psicossociais do trabalho nomeados como protetores (ou seja, que podem reduzir o efeito nocivo de características laborais sobre a saúde) e fatores estressores (aqueles que tendem a produzir ou elevar a nocividade do trabalho sobre a saúde).

Assim, a variável latente relativa aos aspectos psicossociais do trabalho estressores (AE) foi mensurada com base em duas dimensões do JCQ: demanda psicológica ( 0 = baixa; 1 = alta) e demanda física ( 0 = baixa; 1 = alta), juntamente com duas dimensões do modelo ERI: esforço $(0$ = baixo; $1=$ alto) e comprometimento excessivo com o trabalho ( 0 = ausente; 1 = presente). Essas variáveis foram hipotetizadas como aquelas capazes de produzir elevações dos chamados hormônios estressores com efeitos negativos sobre a saúde.

A variável latente aspectos psicossociais do trabalho protetores (AP) foi mensurada com base em duas dimensões do JCQ: controle ( 0 = alto; 1 = baixo); apoio social ( 0 = alto; 1 = baixo $)$ e uma dimensão do ERI: recompensa $(0$ = alta; 1 = baixa).

Por fim, a terceira variável latente, SAP, foi obtida baseando-se em três questões relacionadas à satisfação, pertencentes ao WHOQOL, questionário que avalia a qualidade de vida. São elas: (1) Quão satisfeito/a você está com suas relações pessoais (amigos, parentes, colegas)? (2) Quão satisfeito/a você está consigo mesmo/a? (3) Quão satisfeito/a você está com sua capacidade para o trabalho? Essas questões foram avaliadas em escala do tipo Likert que variou em pontuação de 1 a 5: 1 = muito insatisfeito/a; 
$2=$ insatisfeito/a; 3 = nem insatisfeito/a, nem satisfeito/a; 4 = satisfeito/a; $5=$ muito satisfeito/a. As opções de resposta foram dicotomizadas em insatisfeito/a, para os valores 1, 2 e 3, e satisfeito/a, para os valores 4 e 5 .

Inicialmente, foram estimadas as frequências das características sociodemográficas, do trabalho e as variáveis de interesse do estudo. Em seguida, utilizou-se a Modelagem de Equações Estruturais (MEE), método que engloba técnicas multivariadas de análise de dados, combinando aspectos de regressão múltipla e de análise fatorial para estimar simultaneamente uma série de relações de dependência 26. A MEE é composta pelo modelo de mensuração e pelo modelo estrutural.

$\mathrm{O}$ modelo de mensuração expõe como as variáveis indicadoras expressam os construtos ou variáveis latentes. Essa etapa foi processada via análise fatorial confirmatória (AFC). Por se tratar de eventos de natureza abstrata (aspectos psicossociais e SAP), foram considerados os itens com cargas fatoriais padronizadas significantes e maiores ou iguais a 0,30 27,28. A validade discriminante dos construtos foi verificada pela correlação entre eles - valores menores que 0,90 indicam validade discriminante satisfatória 28 .

O modelo estrutural expressa como os construtos e as variáveis observadas se relacionam, por meio do cálculo de equações simultâneas 26, extraídas das relações teóricas estabelecidas entre os eventos de interesse (Figura 1). No modelo estimado foram avaliados os efeitos diretos e indiretos das variáveis latentes e observadas. Julgou-se haver efeito significante quando $\mathrm{p}<0,05$. Quanto à magnitude do efeito, foi considerado grande para valores em torno de 0,5, efeito médio em torno de 0,3 e efeito pequeno em torno de 0,1 unidades de desvio padrão 29.

Para obter sugestões de alterações das hipóteses iniciais foram avaliados os Índices de Modificação (MI). Diante de valores de MI $\geq 10.000$ e plausibilidade teórica das modificações propostas, um novo modelo foi reespecificado 30 .

Considerando-se as relações de gênero e a divisão sexual do trabalho, com maior vulnerabilidade da população feminina ao trabalho precário e ao adoecimento mental 31 , todas as análises foram estratificadas por gênero.

O software usado para a análise foi o Mplus versão 6 (https://www.statmodel.com/). Foi empregado o método de Médias Ponderadas dos Mínimos Quadrados e Variância Ajustada (Weighted Least Squares Mean and Variance Adjusted - WLSMV), apropriado para as variáveis categóricas, estimando as cargas fatoriais e os coeficientes de regressão brutos e padronizados, com IC95\%.

Para avaliar a adequação dos modelos foram utilizadas medidas de ajustamento, conforme recomendado por Hair et al. 20: índice de ajuste comparativo (Comparative Fit Index - CFI $\geq 0$,90); índice de Tucker-Lewis (TLI $\geq 0,90$ ); e raiz do erro quadrático médio de aproximação (Root Mean Square Error of Approximation - RMSEA < 0,06), e limite superior do intervalo de $90 \%$ de confiança (IC90\%) < 0,08. Para a avaliação dos modelos de mensuração foram avaliadas, ainda, as medidas de Variância Média Extraída (VME > 0,50), Confiabilidade Composta $(\mathrm{CC}>0,70)$ e os respectivos intervalos de $95 \%$ de confiança (IC95\%).

O presente estudo respeitou os requisitos da Resolução no 466/2012 do Conselho Nacional de Saúde, com aprovação do Comitê de Ética em Pesquisa, Universidade Estadual de Feira de Santana, sob o número de protocolo: 081/2009 (CAE 0086.0.059.000-09).

\section{Resultados}

A amostra foi constituída por 3.084 trabalhadores/as da saúde, em sua maioria mulheres (78,2\%). O perfil predominante entre os homens e as mulheres foi: jovens com até 40 anos de idade, negros/as, com nível fundamental ou médio de escolaridade, com companheiro/a, filho/a e renda mensal de até dois salários mínimos. A renda mensal predominante foi de até dois salários mínimos. Agentes comunitários/as de saúde foi o grupo profissional de maior proporção, seguido pela categoria de "outros/ as" que incluiu pessoal administrativo, de nível médio, vigilantes, motoristas e trabalhadores/as de serviços gerais (Tabela 1).

Entre os homens, 27,4\% relataram estar insatisfeitos com o trabalho, percentual discretamente maior do que entre as mulheres, 25,6\%. A prevalência de TMC foi de 17,2\% entre os homens e $23 \%$ entre as mulheres. Destacaram-se elevadas proporções dos indicadores de baixo apoio social e de 


\section{Figura 1}

Modelo estrutural representando os efeitos diretos e indiretos da insatisfação com o trabalho sobre a saúde mental, segundo gênero. Bahia, Brasil, 2011-2012.

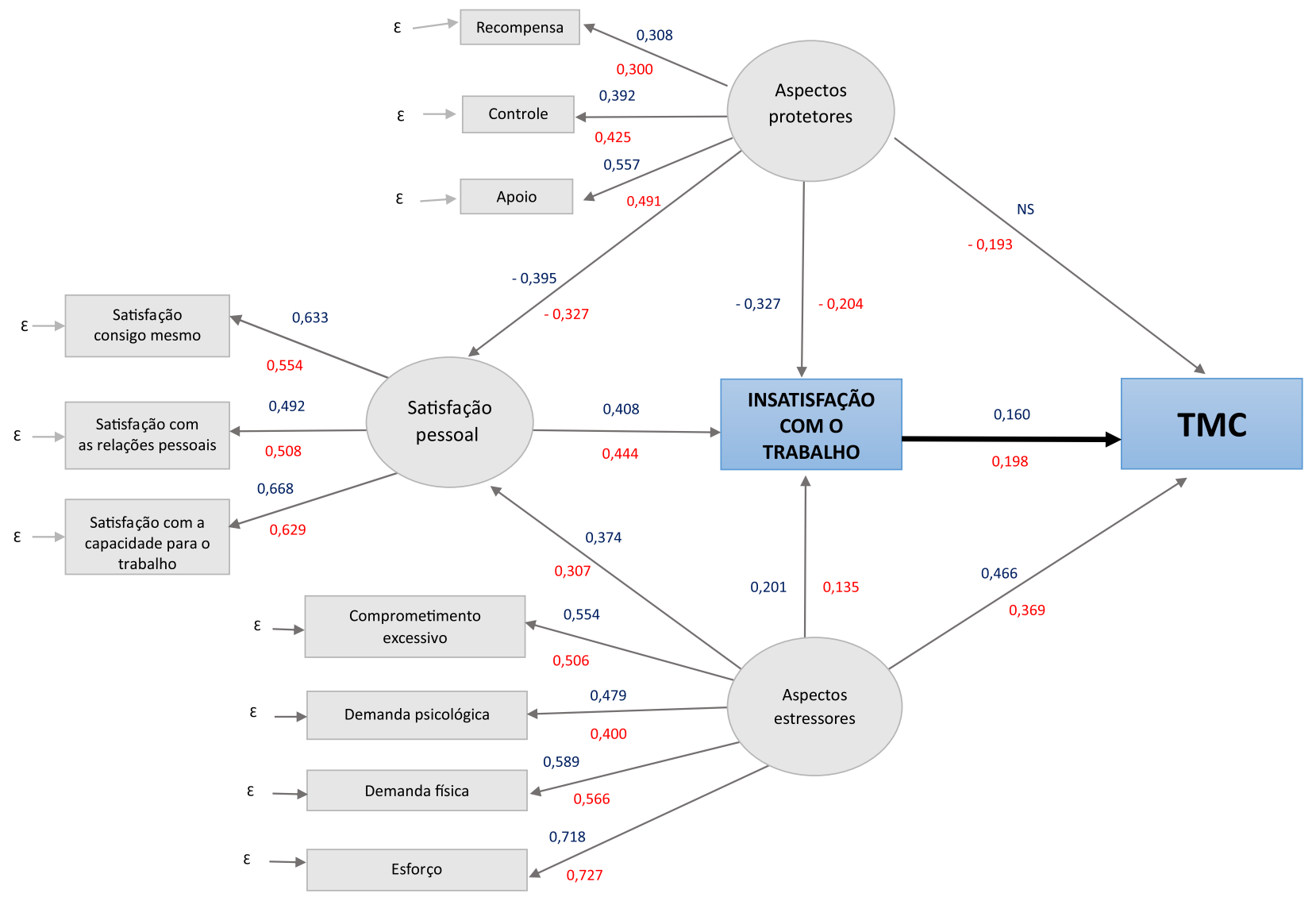

$\varepsilon:$ erro; NS: não significante; TMC: transtorno mental comum.

Nota: os coeficientes em vermelho = valores estimados entre as mulheres; em azul = valores estimados entre homens

baixa recompensa, entre homens e mulheres. As proporções de SAP foram similares entre os estratos. Os homens apresentaram um percentual maior de alta demanda psicológica. As mulheres referiram maiores percentuais de baixo controle, alta demanda física, alto esforço, baixa recompensa e presença de comprometimento excessivo com o trabalho (Tabela 2).

Os modelos de mensuração apresentaram itens com cargas fatoriais aceitáveis $(>0,30)$ e significantes para todos os construtos/variáveis latentes avaliados. Os modelos se ajustaram satisfatoriamente e a validade discriminante foi corroborada (Tabela 3).

O modelo estrutural indicou os efeitos diretos e indiretos das variáveis explicativas sobre os TMC e sobre a insatisfação com o trabalho (Tabela 4 e Figura 1). A insatisfação com o trabalho associouse positivamente com os TMC, sendo estatisticamente significante entre os homens (0,160; IC95\%: 0,024; 0,295) e as mulheres (0,198; IC95\%: 0,135; 0,260).

Os AE associaram-se positivamente à insatisfação com o trabalho, aos TMC, e à SAP, nos estratos masculino e feminino, em níveis estatisticamente significantes. Ou seja, na situação de exposição aos $\mathrm{AE}$ (alta demanda física e psicológica, alto esforço e alto comprometimento excessivo) observaram-se as maiores ocorrências de insatisfação laboral e pessoal e de TMC. 
Tabela 1

Características sociodemográficas e laborais dos/as trabalhadores/as da saúde, segundo gênero. Bahia, Brasil, 2011-2012.

\begin{tabular}{|c|c|c|c|c|}
\hline \multirow[t]{2}{*}{ Características sociodemográficas } & \multicolumn{2}{|c|}{ Mulheres } & \multicolumn{2}{|c|}{ Homens } \\
\hline & $n$ & $\%$ & $n$ & $\%$ \\
\hline \multicolumn{5}{|l|}{ Idade (anos) $[\mathrm{n}=3.058]$ * } \\
\hline Até 40 & 1.384 & 58,0 & 396 & 59,1 \\
\hline Mais de 40 & 1.004 & 42,0 & 274 & 40,9 \\
\hline \multicolumn{5}{|l|}{ Cor da pele autorreferida $[\mathrm{n}=2.984]$ * } \\
\hline Pretos/as e pardos/as & 1.938 & 84,0 & 503 & 78,6 \\
\hline Brancos/as & 370 & 16,0 & 137 & 21,4 \\
\hline \multicolumn{5}{|l|}{ Situação conjugal $[n=3.070]$ * } \\
\hline Com companheiro/a & 1.364 & 56,8 & 393 & 58,7 \\
\hline Sem companheiro/a & 1.037 & 43,2 & 276 & 41,3 \\
\hline \multicolumn{5}{|l|}{ Escolaridade $[n=3.038]$ * } \\
\hline Nível Superior & 645 & 27,1 & 209 & 31,6 \\
\hline Nível Técnico & 452 & 19,1 & 59 & 8,9 \\
\hline Nível Médio e Fundamental & 1.280 & 53,8 & 393 & 59,5 \\
\hline \multicolumn{5}{|l|}{ Tem filhos $[n=3.062]$ * } \\
\hline Sim & 1.677 & 70,1 & 428 & 63,9 \\
\hline Não & 715 & 29,9 & 242 & 36,1 \\
\hline \multicolumn{5}{|l|}{ Renda (salários mínimos) [n = 2.556] * } \\
\hline Até 2 & 1.464 & 73,8 & 339 & 59,3 \\
\hline Mais de 2 & 520 & 26,2 & 233 & 40,7 \\
\hline \multicolumn{5}{|l|}{ Categoria profissional $[n=3.068]$ * } \\
\hline Enfermeiro/a & 188 & 7,9 & 25 & 3,7 \\
\hline Médico/a & 68 & 2,9 & 78 & 11,7 \\
\hline Outros/as de Nível Superior & 169 & 7,1 & 59 & 8,9 \\
\hline Técnico/a de enfermagem & 387 & 16,2 & 25 & 3,8 \\
\hline Agente comunitário de saúde & 804 & 33,5 & 126 & 19,0 \\
\hline Outros/as categorias & 784 & 32,4 & 355 & 52,9 \\
\hline
\end{tabular}

* Os n variaram em função de perdas de informações para as variáveis analisadas.

Observaram-se associações negativas entre os AP e a insatisfação com o trabalho, a satisfação pessoal e os TMC, entre homens e mulheres. Assim, na presença dos aspectos psicossociais protetores (alto controle, alta recompensa e alto apoio social) observaram-se menores ocorrências de insatisfação laboral e pessoal e de TMC. Contudo, a associação entre os AP e os TMC apresentou significância estatística apenas no estrato feminino (-0,193; IC95\%: -0,285; -0,101).

Os aspectos psicossociais do trabalho estavam associados à ocorrência de TMC, mesmo quando mediados pela insatisfação com o trabalho, em níveis estatisticamente significantes, nos estratos de gênero considerados. Os efeitos indiretos dos AP e AE sobre os TMC foram menores do que o efeito direto. Os AP continuaram relacionando-se negativamente com os TMC, apesar da mediação do efeito pela insatisfação com o trabalho.

A SAP exerceu efeito positivo indireto sobre os TMC, com significância entre as mulheres $(0,088$; IC95\%: 0,053; 0,123). Os modelos de mensuração e estrutural estimados apresentaram índices de ajustes adequados entre os homens (CFI =0,952; TLI =0,933; RMESA =0,040) e entre as mulheres $(\mathrm{CFI}=0,915 ; \mathrm{TLI}=0,900 ; \mathrm{RMESA}=0,053)$. 
Tabela 2

Distribuição da insatisfação com o trabalho, transtornos mentais comuns, aspectos psicossociais do trabalho e características da satisfação pessoal entre trabalhadores/as da saúde, segundo gênero. Bahia, Brasil, 2011-2012.

\begin{tabular}{|c|c|c|c|c|}
\hline \multirow[t]{2}{*}{ Variáveis } & \multicolumn{2}{|c|}{ Mulheres } & \multicolumn{2}{|c|}{ Homens } \\
\hline & $\mathbf{n}$ & $\%$ & $\mathbf{n}$ & $\%$ \\
\hline \multicolumn{5}{|c|}{ Insatisfação com o trabalho $[\mathrm{n}=3.072]$ * } \\
\hline Não & 1.788 & 74,4 & 487 & 72,6 \\
\hline $\operatorname{Sim}$ & 615 & 25,6 & 184 & 27,4 \\
\hline \multicolumn{5}{|c|}{ Transtornos mentais comuns $[\mathrm{n}=2.999]$ * } \\
\hline Não & 1.806 & 77,0 & 541 & 82,8 \\
\hline $\operatorname{Sim}$ & 540 & 23,0 & 112 & 17,2 \\
\hline \multicolumn{5}{|c|}{ Demanda psicológica $[\mathrm{n}=3.023]$ * } \\
\hline Baixa & 1.366 & 57,9 & 346 & 52,1 \\
\hline Alta & 993 & 42,1 & 318 & 47,9 \\
\hline \multicolumn{5}{|c|}{ Controle sobre o trabalho $[n=2.932]$ * } \\
\hline Alto & 1.143 & 49,9 & 355 & 55,3 \\
\hline Baixo & 1.147 & 50,1 & 287 & 44,7 \\
\hline \multicolumn{5}{|c|}{ Demanda física $[n=3.043]$ * } \\
\hline Baixa & 1.542 & 64,8 & 481 & 72,4 \\
\hline Alta & 837 & 35,2 & 183 & 27,6 \\
\hline \multicolumn{5}{|c|}{ Apoio social no trabalho $[n=2.861]$ * } \\
\hline Alto & 650 & 28,7 & 171 & 28,8 \\
\hline Baixo & 1.617 & 71,3 & 423 & 71,2 \\
\hline \multicolumn{5}{|c|}{ Esforço $[n=3.045]$ * } \\
\hline Baixo & 1.624 & 68,3 & 471 & 70,6 \\
\hline Alto & 754 & 31,7 & 196 & 29,4 \\
\hline \multicolumn{5}{|c|}{ Recompensa $[n=3.011]$ * } \\
\hline Alta & 692 & 29,3 & 216 & 33,2 \\
\hline Baixa & 1.669 & 70,7 & 434 & 66,8 \\
\hline \multicolumn{5}{|c|}{ Comprometimento excessivo com o trabalho $[n=3.037]$ * } \\
\hline Ausente & 1.272 & 53,6 & 393 & 59,3 \\
\hline Presente & 1.102 & 46,4 & 270 & 40,7 \\
\hline \multicolumn{5}{|c|}{ Satisfação consigo mesmo $[n=3.067]$ * } \\
\hline Satisfeito/a & 2.058 & 85,7 & 569 & 85,2 \\
\hline Insatisfeito/a & 341 & 14,2 & 99 & 14,8 \\
\hline \multicolumn{5}{|c|}{ Satisfação com suas relações pessoais $[n=3.066]$ * } \\
\hline Satisfeito/a & 2.077 & 86,6 & 568 & 85,0 \\
\hline Insatisfeito/a & 321 & 13,4 & 100 & 15,0 \\
\hline \multicolumn{5}{|c|}{ Satisfação com a capacidade para o trabalho $[n=3.069]$ * } \\
\hline Satisfeito/a & 1.841 & 76,7 & 501 & 74,9 \\
\hline Insatisfeito/a & 559 & 23,3 & 168 & 25,1 \\
\hline
\end{tabular}

* Os n variaram em função de perdas de informações para as variáveis analisadas. 
Tabela 3

Modelo de mensuração das variáveis latentes de trabalhadores/as da saúde, segundo gênero. Bahia, Brasil, $2011-2012$.

\begin{tabular}{|c|c|c|}
\hline \multirow[t]{2}{*}{ Modelos de mensuração } & \multicolumn{2}{|c|}{ Estimativas (IC95\%) } \\
\hline & Homens & Mulheres \\
\hline \multicolumn{3}{|l|}{ Satisfação pessoal (SAP) } \\
\hline Satisfação consigo mesmo & $0,633(0,553 ; 0,713)$ & $0,554(0,507 ; 0,601)$ \\
\hline Satisfação com as relações pessoais & $0,492(0,407 ; 0,577)$ & $0,508(0,460 ; 0,555)$ \\
\hline Satisfação com a capacidade para o trabalho & $0,668(0,593 ; 0,742)$ & $0,629(0,581 ; 0,677)$ \\
\hline \multicolumn{3}{|l|}{ Aspectos estressores (AE) } \\
\hline Comprometimento excessivo & $0,554(0,491 ; 0,617)$ & $0,506(0,469 ; 0,542)$ \\
\hline Demanda psicológica & $0,479(0,411 ; 0,546)$ & $0,400(0,360 ; 0,441)$ \\
\hline Demanda física & $0,589(0,528 ; 0,650)$ & $0,566(0,531 ; 0,602)$ \\
\hline Esforço & $0,718(0,659 ; 0,777)$ & $0,727(0,694 ; 0,760)$ \\
\hline \multicolumn{3}{|l|}{ Aspectos protetores (AP) } \\
\hline Recompensa & $0,308(0,193 ; 0,422)$ & $0,300(0,229 ; 0,360)$ \\
\hline Controle & $0,392(0,273 ; 0,512)$ & $0,425(0,411 ; 0,571)$ \\
\hline Apoio & $0,557(0,415 ; 0,699)$ & $0,491(0,350 ; 0,500)$ \\
\hline \multicolumn{3}{|l|}{ Ajuste do modelo } \\
\hline RMSEA & $0,040(0,035 ; 0,046)$ & $0,053(0,047 ; 0,060)$ \\
\hline $\mathrm{CFI}$ & 0,952 & 0,915 \\
\hline TLI & 0,933 & 0,900 \\
\hline \multicolumn{3}{|l|}{ Variância média extraída (VME) } \\
\hline SAP & $0,63(0,55 ; 0,71)$ & $0,58(0,54 ; 0,63)$ \\
\hline $\mathrm{AE}$ & $0,55(0,50 ; 0,60)$ & $0,49(0,46 ; 0,52)$ \\
\hline AP & $0,34(0,04 ; 0,63)$ & $0,38(0,16 ; 0,56)$ \\
\hline \multicolumn{3}{|l|}{ Confiabilidade composta (CC) } \\
\hline SAP & $0,84(0,79 ; 0,88)$ & $0,81(0,78 ; 0,83)$ \\
\hline $\mathrm{AE}$ & $0,83(0,79 ; 0,86)$ & $0,79(0,77 ; 0,81)$ \\
\hline $\mathrm{AP}$ & $0,57(0,35 ; 0,79)$ & $0,59(0,44 ; 0,72)$ \\
\hline
\end{tabular}

CFI: índice de ajuste comparativo; TLI: índice de Tucker-Lewis; RMSEA: raiz do erro quadrático médio de aproximação.

\section{Discussão}

A insatisfação com o trabalho esteve associada aos TMC entre homens e mulheres, com medidas de associação de maior magnitude entre as mulheres. Os AP associaram-se à menor ocorrência da insatisfação com o trabalho e de TMC, e os AE estiveram associados à maior frequência de insatisfação e de TMC, em ambos os grupos. A insatisfação com o trabalho mediou o efeito dos aspectos psicossociais e da SAP na ocorrência de TMC.

Os resultados encontrados corroboram o conhecimento descrito na literatura: o impacto negativo da insatisfação com o trabalho na saúde mental 1,2,3,7; a repercussão do estresse ocupacional na insatisfação com o trabalho 1,32,33; bem como a associação entre estressores ocupacionais e TMC 12. Também fortalecem as duas hipóteses de trabalho investigadas: (1) associação entre a insatisfação e transtornos mentais (a insatisfação contribui diretamente para a ocorrência de TMC); (2) os aspectos psicossociais do trabalho (estressores e protetores) e a SAP podem associar-se positiva ou negativamente tanto à insatisfação quanto aos transtornos mentais, modificando a relação entre a insatisfação com o trabalho e os TMC.

Elevados percentuais de insatisfação com o trabalho em saúde, entre homens e mulheres, revelam vivência frequente de situações desgastantes no ambiente laboral. A organização e a divisão do trabalho apresentam-se, muitas vezes, alheia à atividade mental do/a trabalhador/a, assim como aos seus desejos e aspirações. Na busca por equilibrar sua saúde psíquica e manter a integridade mental, 
Coeficientes padronizados (CP) e intervalo de $95 \%$ de confiança (IC95\%) do modelo de equações estruturais utilizandose transtornos mentais comuns (TMC) como variável desfecho, em trabalhadores/as da saúde, segundo gênero. Bahia, Brasil, 2011-2012.

\begin{tabular}{|c|c|c|c|c|}
\hline \multirow[t]{3}{*}{ Modelo estrutural } & \multicolumn{4}{|c|}{ Coeficientes padronizados } \\
\hline & \multicolumn{2}{|c|}{ Homens } & \multicolumn{2}{|c|}{ Mulheres } \\
\hline & $\mathbf{C P}$ & IC95\% & CP & IC95\% \\
\hline \multicolumn{5}{|l|}{ Efeitos diretos } \\
\hline $\mathrm{AE} \rightarrow \mathrm{SAP}$ & 0,374 & 0,$255 ; 0,493$ & 0,307 & 0,$244 ; 0,370$ \\
\hline $\mathrm{AP} \rightarrow \mathrm{SAP}$ & $-0,395$ & $-0,560 ;-0,229$ & $-0,327$ & $-0,410 ;-0,244$ \\
\hline $\mathrm{SAP} \rightarrow$ Insatisfação & 0,408 & 0,$255 ; 0,560$ & 0,444 & 0,$366 ; 0,521$ \\
\hline $\mathrm{AE} \rightarrow$ Insatisfação & 0,201 & 0,$060 ; 0,341$ & 0,135 & 0,$067 ; 0,203$ \\
\hline $\mathrm{AP} \rightarrow$ Insatisfação & $-0,327$ & $-0,515 ;-0,138$ & $-0,204$ & $-0,299 ;-0,109$ \\
\hline $\mathrm{AE} \rightarrow \mathrm{TMC}$ & 0,466 & 0,$318 ; 0,614$ & 0,369 & 0,$304 ; 0,434$ \\
\hline $\mathrm{AP} \rightarrow \mathrm{TMC}$ & $-0,079$ & $-0,295 ; 0,138$ * & $-0,193$ & $-0,285 ;-0,101$ \\
\hline Insatisfação $\rightarrow$ TMC & 0,160 & 0,$024 ; 0,295$ & 0,198 & 0,$135 ; 0,260$ \\
\hline \multicolumn{5}{|l|}{ Efeitos indiretos } \\
\hline $\mathrm{SAP} \rightarrow$ Insatisfação $\rightarrow$ TMC & 0,065 & $-0,001 ; 0,131$ & 0,088 & 0,$053 ; 0,123$ \\
\hline $\mathrm{AE} \rightarrow$ Insatisfação $\rightarrow \mathrm{TMC}$ & 0,032 & 0,$002 ; 0,062$ & 0,027 & 0,$012 ; 0,041$ \\
\hline $\mathrm{AP} \rightarrow$ Insatisfação $\rightarrow$ TMC & $-0,052$ & $-0,100 ; 0,004$ & $-0,040$ & $-0,060 ;-0,021$ \\
\hline \multicolumn{5}{|l|}{ Ajuste do modelo } \\
\hline RMSEA & \multicolumn{2}{|c|}{$0,040(0,035 ; 0,046)$} & \multicolumn{2}{|c|}{$0,053(0,047 ; 0,060)$} \\
\hline CFI & \multicolumn{2}{|c|}{0,952} & \multicolumn{2}{|c|}{0,915} \\
\hline TLI & \multicolumn{2}{|c|}{0,933} & \multicolumn{2}{|c|}{0,900} \\
\hline
\end{tabular}

AE: aspectos estressores; AP: aspectos protetores; CFI: índice de ajuste comparativo; RMSEA: raiz do erro quadrático médio de aproximação; SAP: satisfação pessoal; TLI: índice de Tucker-Lewis.

* Estimativas não significantes.

o/a trabalhador/a estabelece estratégias para adaptar as suas competências e modelar as motivações ao trabalho. Contudo, grandes esforços podem não ser suficientes na tentativa de manter a satisfação com o trabalho, emergindo, assim, o sofrimento e a insatisfação 34 .

A inserção em um ambiente de trabalho repleto de cargas psíquicas repercute na saúde mental dos/as trabalhadores/as, a exemplo das elevadas prevalências de TMC verificadas nesta população, sendo as mulheres o grupo mais afetado, com maior prevalência de TMC do que os homens, como identificado em outros estudos no setor saúde 11,13. Evidenciou-se, ainda, maior exposição feminina ao adoecimento mental, quando insatisfeitas com o trabalho.

Neste estudo, verificou-se intensa feminização do trabalho em saúde, em conformidade com a literatura ${ }^{35}$. Isso decorre do processo de divisão sexual do trabalho, em que as habilidades e qualificações ensinadas às mulheres no âmbito privado das famílias são reproduzidas nas organizações 36 , como atividades voltadas para ações de cuidado, por exemplo. Dessa maneira, as mulheres estão mais inseridas nas atividades com menor remuneração e reconhecimento social. Essas características e condições laborais, por sua vez, têm sido consistentemente associadas à morbidade psíquica ${ }^{31}$, sendo fatores que contribuem para a maior ocorrência de TMC.

Os aspectos psicossociais influenciaram diretamente os níveis de insatisfação com o trabalho e de ocorrência de TMC entre homens e mulheres. A variável latente $\mathrm{AE}$ esteve associada positivamente à insatisfação e aos TMC, confirmando a nocividade das dimensões deste construto (elevados níveis de demanda psicológica, demanda física, esforço e comprometimento excessivo com o trabalho) sobre os níveis de satisfação laboral e também sobre a saúde mental dos/as trabalhadores/as.

Estudos prévios observaram que as demandas físicas e psicológicas do trabalho, variáveis indicadoras do construto $\mathrm{AE}$, quando elevadas, contribuem para o aumento do estresse ocupacional com repercussão nos níveis de satisfação com o trabalho 1,33 e na saúde mental 12. 
O esforço refere-se à resposta do indivíduo às demandas que lhe são apresentadas. Quando o esforço com o trabalho não é recompensado adequadamente, contribui para elevar o grau de insatisfação com o trabalho. O desequilíbrio entre os esforços despendidos e as recompensas recebidas resulta em efeitos negativos sobre a saúde. Pressupõe-se que altos esforços seguidos por baixas recompensas desencadeiam emoções negativas e respostas sustentadas de estresse elevado, já o equilíbrio entre os esforços e as recompensas gera emoções positivas capazes de promover bem-estar e saúde no trabalho 37 .

$\mathrm{O}$ comprometimento excessivo com o trabalho refere-se à necessidade de se manter o tempo todo vinculado às situações de trabalho. Nessa perspectiva, o/a trabalhador/a se envolve demasiadamente com o trabalho, com maior dispêndio de energia ${ }^{37}$. Em geral, nessa situação, a pessoa eleva muito as expectativas com o trabalho, dependendo excessivamente de reconhecimento. A ausência desse reconhecimento pode produzir elevados níveis de insatisfação com o trabalho.

Os AP, variável latente estimada com base em dimensões de apoio social, controle e recompensa, associaram-se negativamente à insatisfação com o trabalho e aos TMC, indicando menor ocorrência de insatisfação laboral e de adoecimento mental quando estes aspectos estavam presentes. A maneira como cada indivíduo vivencia esses aspectos pode elevar os níveis de estresse ou moderar/reduzir os níveis de estresse ocupacional sobre a insatisfação com o trabalho e a saúde mental do/a trabalhador/a.

O apoio social proveniente da chefia e dos colegas de trabalho tem papel primordial nos níveis de satisfação. Quando o estilo de liderança dos supervisores envolve a preocupação com o auxílio ao/à trabalhador/a no desenvolvimento de suas atividades, verifica-se maior envolvimento e percepção positiva do trabalho. O apoio proveniente dos colegas é mais decisivo em ocupações de trabalho em equipe, a exemplo dos serviços de saúde 38 . Essa dimensão estimula a sensação de pertencimento ao grupo e eleva o envolvimento com o trabalho. Quando existe tal apoio, o ambiente laboral torna-se mais agradável e os/as trabalhadores/as sentem-se mais satisfeitos, ainda que demandas elevadas estejam presentes 38 . Nossos resultados corroboram esse pressuposto de redução da insatisfação na presença de AP.

O controle sobre o próprio trabalho esteve associado inversamente à insatisfação. Quando o/a trabalhador/a exerce controle sobre a atividade que executa, seja na utilização do tempo, na definição dos modos operatórios ou das ferramentas de trabalho, podem ser estabelecidas importantes estratégias de luta contra o sofrimento e o adoecimento. A possibilidade de adaptar o trabalho aos anseios e modos particulares dos/as trabalhadores/as favorece a construção de subjetividades vinculadas à atividade profissional o que, consequentemente, pode reduzir a insatisfação com o trabalho ${ }^{34}$. Ou seja, o exercício de controle sobre o próprio trabalho pode possibilitar ao/à trabalhador/a modelar as exigências do trabalho às suas capacidades e habilidades; ao exercer sua autonomia, pode alterar as condições gerais do trabalho, ampliando aspectos positivos e reduzindo os efeitos nocivos causados pelas demandas excessivas.

As recompensas obtidas com o trabalho equalizam o desgaste causado pelo esforço elevado e constituem fonte de satisfação ${ }^{39}$. Ressalta-se que, no trabalho em saúde, as recompensas são expressas não somente de forma material (como o salário), mas também de maneira imaterial, como a possibilidade de interferir na situação de saúde de uma pessoa, o contato com diferentes realidades, a troca de afeto, o sentimento de confiança e a gratidão por parte dos usuários são fontes de recompensa 12.

Os estressores laborais influenciaram a SAP, confirmando a relevância dos aspectos psicossociais do trabalho sobre a vida extra laboral do/a trabalhador/a. A SAP associou-se à insatisfação com o trabalho e, indiretamente, com os TMC, quando mediada pela insatisfação com o trabalho.

Embora o fato de possuir um emprego não seja garantia de realização profissional e pessoal, uma vez que o ambiente do trabalho não é neutro e traz consigo situações diversas, algumas vezes desfavoráveis à saúde do/a trabalhador/a 40 , observa-se que o trabalho desempenha um papel relevante na SAP, como apontam nossos resultados. As experiências laborais, assim, podem produzir situações nocivas que extrapolam o ambiente de trabalho, com repercussões nos comportamentos individuais e nas relações pessoais. A insatisfação pessoal, em parte associada aos estressores ocupacionais, é uma carga psíquica adicional e se relaciona positivamente com a insatisfação com o trabalho. Dado que essa insatisfação decorre também de fontes externas ao ambiente laboral, pode-se considerar que a insatisfação pessoal também afeta negativamente os níveis de satisfação com o trabalho.

Um estudo realizado com médicos intensivistas estimou associação positiva e significante entre a SAP e profissional 16. O apoio da família e dos amigos permite ao/a trabalhador/a uma vida mais 
equilibrada, resultando em sentimentos positivos e maior SAP, elevando o envolvimento e a satisfação com o trabalho 41 .

Os achados deste estudo reforçam os resultados de associação da insatisfação com o trabalho com o adoecimento mental e a associação dos aspectos psicossociais com a insatisfação e com os TMC. A principal vantagem deste estudo reside no fato de os efeitos diretos e indiretos de cada variável serem estimados simultaneamente, contribuindo para a melhor compreensão destas relações. Dessa maneira, a insatisfação laboral relaciona-se com o adoecimento mental, apresentando associação direta e positiva com os TMC e mediando o efeito dos estressores laborais. Contudo, a falta de estudos similares dificultou a comparação dos resultados obtidos com a literatura. Ademais, a limitação proveniente dos dados analisados serem de desenho de estudo pontual no tempo também impõe barreiras a explorações mais detalhadas de sequência temporal e causalidade. Deve-se considerar, contudo, que os resultados obtidos fortalecem as hipóteses iniciais pressupostas, favorecendo explorações futuras com estudos longitudinais.

Neste estudo, a evidência de que a insatisfação com o trabalho pode ser mediadora dos efeitos dos estressores ocupacionais sobre a saúde mental constitui uma contribuição importante para políticas no campo as saúde do/a trabalhador/a. Para a saúde pública, esses achados representam ganhos importantes, uma vez que podem nortear a adoção de estratégias que incidam diretamente sobre a exposição, a fim de elevar os níveis de satisfação com o trabalho, favorecendo a manutenção da saúde mental.

Nesse sentido, recomenda-se considerar a adoção de estratégias de enfrentamento que reduzam os aspectos estressores do trabalho (como, por exemplo, dimensionamento adequado de pessoal, condições salubres de trabalho, redução da carga horária de trabalho semanal, adoção de medidas coletivas de proteção), que elevem as dimensões protetoras (tais como liderança participativa, tomada de decisões compartilhada, estímulo à autonomia sobre o trabalho, implementação de planos de carreira, cargos e salários, cumprimento dos direitos trabalhistas) e que considerem os diferenciais de gênero no que se refere aos níveis de insatisfação com o trabalho e ao adoecimento mental relacionado ao trabalho. Essas medidas, se adotadas, podem contribuir na redução dos níveis de insatisfação e dos transtornos mentais relacionados ao trabalho, promovendo, assim, ambientes de trabalho mais saudáveis.

Por fim, é preciso considerar as limitações inerentes aos estudos transversais. Exposição e desfecho foram analisados em um único momento do tempo, não podendo ser descartada a possibilidade de causalidade reversa. $\mathrm{O}$ viés de prevalência, situação na qual os indivíduos que possuem o desfecho por maior período de tempo têm maior chance de serem incluídos na pesquisa; o viés do trabalhador sadio, dado que foram entrevistados/as apenas os/as trabalhadores/as que estavam em pleno exercício de suas atividades, não sendo incluídos/as os/as afastados/as por problemas de saúde; e o viés de memória, ao qual o desfecho está vulnerável, visto que sua estimação depende do resgate de informações passadas.

Destacamos que o emprego de MEE para a análise de desfechos em saúde revelou-se uma ferramenta potente para ampliar o entendimento da complexidade dos eventos em saúde mental, com a possibilidade de avaliar não apenas a associação entre uma exposição e um desfecho, como também avaliar as relações entre as diferentes exposições e seus efeitos diretos ou mediados sobre a saúde. Ressalta-se a necessidade de investimento em métodos epidemiológicos e estatísticos de análises avançados como esforço para capturar a multicausalidade que determina as situações de saúde/doença, incorporando a inter-relação entre os fatores, de modo mais aproximado a como eles ocorrem em contextos concretos. 


\section{Colaboradores}

C. C. Sousa contribuiu na concepção e design, aquisição, análise e interpretação dos dados, redigiu o artigo e revisou criticamente, aprovou a versão final para publicação, e é responsável por todos os aspectos do trabalho. T. M. Araújo, I. Lua, M. R. Gomes e K. S. Freitas contribuíram na análise e interpretação de dados, revisão do artigo, aprovação final da versão a ser publicada, são responsáveis por todos os aspectos do trabalho.

\section{Informações adicionais}

ORCID: Camila Carvalho de Sousa (0000-00026139-0984); Tânia Maria de Araújo (0000-00032766-7799); Iracema Lua (0000-0002-5760-5073); Mariana Rabelo Gomes (0000-0001-9875-7308); Kátia Santana Freitas (0000-0002-0491-6759).

\section{Agradecimentos}

À Fundação de Amparo à Pesquisa do Estado da Bahia e à Coordenação de Aperfeiçoamento de Pessoal de Nível Superior pelo financiamento.

\section{Referências}

1. Ribeiro RBN, Assunção AA, Araújo TM. Factors associated with job satisfaction among public-sector physicians in Belo Horizonte, Brazil. Int J Health Serv 2014; 44:787-804.

2. Lu H, Barriball KL, Zhang X, While AE. Job satisfaction among hospital nurses revisited: a systematic review. Int J Nurs Stud 2012; 49:1017-38.

3. Kwaka C, Chungb, BY, Xu Y, Eun-Jungd C. Relationship of job satisfaction with perceived organizational support and quality of care among South Korean nurses: a questionnaire survey. Int J Nurs Stud 2010; 47:1292-8.

4. Melo MB, Barbosa MA, Souza PR. Job satisfaction of nursing staff: integrative review. Rev Latinoam Enferm 2011; 19:1047-55.

5. Lapischies SRC, Jardim VMR, Kantorski LP. Fatores associados à satisfação no trabalho em Centros de Atenção Psicossocial. Rev Latinoam Enferm 2014; 22:950-8.

6. Tarcan M, Hikmet N, Schooley B, Top M, Tarcan GY. An analysis of the relationship between burnout, socio-demographic and workplace factors and job satisfaction among emergency department health professionals. Appl Nurs Res 2017; 34:40-7.

7. Assunção AÁ, Pimenta AM. Satisfação no trabalho do pessoal de enfermagem na rede pública de saúde em uma capital brasileira. Ciênc Saúde Colet 2020; 25:169-80.

8. Goldberg DP, Huxley PJ. Common mental disorders: a bio-social model. London: Tavistock/ Routledge; 1992.

9. Braga LC, Carvalho LR, Binder MCP. Condições de trabalho e transtornos mentais comuns em trabalhadores da rede básica de saúde de Botucatu - SP. Ciênc Saúde Colet 2010; 15 Suppl 1:1585-96.

10. Silva-Junior JS, Fischer FM. Afastamento do trabalho por transtornos mentais e estressores psicossociais ocupacionais. Rev Bras Epidemiol 2015; 18:735-44.

11. Santos FF, Brito MFSF, Pinho L, Cunha F, Rodrigues Neto JF, Fonseca ADG, et al. Common mental disorders in nursing technicians of a university hospital. Rev Bras Enferm 2020; 73:e20180513.

12. Araújo TM, Mattos AIS, Almeida MMG, Santos KOB. Psychosocial aspects of work and common mental disorders among health workers: contributions of combined models. Rev Bras Epidemiol 2016; 19:645-57.

13. Alves A, Pedrosa L, Coimbra M, Miranzi M, Hass V. Prevalência de transtornos mentais comuns entre profissionais de saúde. Rev Enferm UERJ 2015; 23:64-9.

14. Knuth BS, Silva RA, Oses JP, Radtke VA, Cocco RA, Jansen K. Mental disorders among health workers in Brazil. Ciênc Saúde Colet 2015; 20:2481-8.

15. Lima L, Pires DEP, Forte ECN, Medeiros F. Satisfação e insatisfação no trabalho de profissionais de saúde da atenção básica. Esc Anna Nery Rev Enferm 2014; 18:17-24. 
16. Fernandes JS, Miranzi SSC, Iwamoto HH, Tavares DMS, Santos CB. A relação dos aspectos profissionais na qualidade de vida dos enfermeiros das equipes Saúde da Família. Rev Esc Enferm USP 2012; 46:404-12.

17. Wanous JP, Reichers AE, Hudy MJ. Overall job satisfaction: how good are single-item measures? J Appl Psychol 1997; 82:247-52.

18. Ommen O, Driller E, Köhler T, Kowalski C, Ernstmann N, Neumann M, et al. The relationship between social capital in hospitals and physician job satisfaction. BMC Health Serv Res 2009; 16:81.

19. Santos KOB, Araújo T M, Pinho OS, Silva ACC. Avaliação de um instrumento de mensuração de morbidade psíquica: estudo de validação do self-reporting questionnaire (SRQ-20). Rev Baiana Saúde Pública 2010; 34:544-60.

20. Hair JF, Black WC, Babin BJ, Anderson RE, Tatham RL. Análise multivariada de dados. 6a Ed. Porto Alegre: Bookman; 2009.

21. Karasek Jr, RA. Job demands, job decision latitude, and mental strain: implications for job redesign. Adm Sci Q 1979; 24:285-308.

22. Siegrist J. Adverse health effects of high- effort - low-reward conditions at work. J Occup Health Psychol 1996; 1:27-41.

23. Pinhatti EDG, Ribeiro RP, Soares MH, Martins JT, Lacerda MR, Galdino MJQ. Aspectos psicossociais do trabalho e distúrbios psíquicos menores na enfermagem: uso de modelos combinados. Rev Latinoam Enferm 2018; 26:e3068.

24. Yu SF, Nakata A, Gu GZ, Swanson NG, Zhou $\mathrm{WH}, \mathrm{He} \mathrm{LH}$, et al. Co-effect of demand-control-support model and effort-reward imbalance model on depression risk estimation in humans: findings from Henan Province of China. Biomed Environ Sci 2013; 26:962-71.

25. Griep RH, Rotenberg L, Landsbergis P, Vasconcellos-Silva PR. Uso combinado de modelos de estresse no trabalho e a saúde autorreferida na enfermagem. Rev Saúde Pública 2011; 45:145-52.

26. Amorim LDAF, Fiaccone RL, Santos CAST, Santos TN, Moraes LTLP, Oliveira NF, et al. Structural equation modeling in epidemiology. Cad Saúde Pública 2010; 26:2251-62.

27. Laros JA. O uso da análise fatorial: algumas diretrizes para pesquisadores. In: Pasquali L, organizador. Análise fatorial para pesquisadores. Brasília: LabPAM Saber e Tecnologia; 2014. p. 141-60.
28. Wang J, Wang X. Structural equation modeling: applications using Mplus. Noida: Thomson Digital; 2012.

29. Kline RB. Principles and practice of structural equation modeling. New York: The Guilford Press; 2015.

30. Brown TA. Methodology in the social sciences. Confirmatory factor analysis for applied research. 2nd Ed. New York: Guilford Press; 2015.

31. Quinn MM, Smith PM. Gender, work, and health. Ann Work Expo Health 2018; 62:38992.

32. Adib IM, Abdul AA, Suhaili NA, Daud AZ, Naing L, Rahman HA. A study into psychosocial work stressors and health care productivity. Int J Occup Environ Med 2019; 10:185-93.

33. Sousa CC, Araújo TM, Lua I, Gomes MR. Occupational stress and job dissatisfaction with health work. Psicol Reflex Crit 2019; 32:18.

34. Dejours C. A loucura do trabalho: estudo sobre a psicopatologia do trabalho. 5a Ed. São Paulo: Editora Cortez; 1992.

35. Bacha AM, Grassioto OR, Cacique DB, Carvasan, GAF, Machado HC. Satisfação no trabalho no contexto hospitalar: uma análise segundo o gênero. Esc Anna Nery Rev Enferm 2015; 19:549-56.

36. Barbosa RHS. Gender and healthcare work: a critical view of community health agents' work. Interface (Botucatu) 2012; 16:751-65.

37. Siegrist J, Wahrendorf M. Work stress and health in a globalized economy: the model of 'effort' - 'reward' imbalance. Cham: Springer; 2016.

38. Alegre I, Mas-Machuca M, Berbegal-Mirabent J. Antecedents of employee job satisfaction: Do they matter? J Bus Res 2016; 69:1390-5.

39. Jonge J, Bosma H, Peter R, Siegrist J. Job strain, effort-reward imbalance and employee wellbeing: a large-scale cross-sectional study. Soc Sci Med 2000; 50:1317-27.

40. Machado MC, Silvestre RC, Kara-José N, Kara-Júnior N. Avaliação da satisfação profissional de funcionários em um hospital público de reconhecida efetividade assistencial. Rev Bras Oftalmol 2014; 73:143-7.

41. Lambert EG, Minor KI, Wells JB, Hogan NL. Social support's relationship to correctional staff job stress, job involvement, job satisfaction, and organizational commitment. Soc Sci J 2016; 53:22-32. 


\section{Abstract}

The objective was to evaluate the relationship between job dissatisfaction and the occurrence of common mental disorders (CMD), considering the psychosocial aspects of work and personal satisfaction. Cross-sectional study, with a sample of 3,084 health workers from five municipalities in Bahia. Structural equation modeling analysis, stratified by gender, was used. Dissatisfaction with work was the main exposure variable. The CMD, the outcome variable, was assessed by the Self-Reporting Questionnaire (SRQ-20). Personal satisfaction (SAP), assessed by World Health Organization Quality of Life (WHOQOL) questions, and stressful (AE) and protective (AP) psychosocial aspects, measured by the Job Content Questionnaire (JCQ) and the Effort-Reward Imbalance scale (ERI), were treated as latent variables. Dissatisfaction with work was associated with higher CMD prevalence, among men (0.160; CI95\%: 0.024; 0.295) and women (0.198; CI95\%: 0.135; 0.260 ). AEs were associated with greater dissatisfaction and higher CMD prevalence, while APs were associated with lower dissatisfaction and lower CMD prevalence, among men and women. Dissatisfaction with work mediated the effect of psychosocial aspects and personal satisfaction on the occurrence of CMD. The final model showed good adjustment. High job dissatisfaction, stressful psychosocial aspects, lack of protective aspects, and personal dissatisfaction were associated directly or indirectly with $C M D$. The results reinforce the need for actions to protect mental health at work by redesigning the harmful aspects identified and strengthening the aspects associated with the lower occurrence of CMD.

Occupational Stress; Job Satisfaction; Mental Disorders

\section{Resumen}

El objetivo fue evaluar la relación de la insatisfacción en el trabajo y la ocurrencia de trastornos mentales comunes (TMC), considerando aspectos psicosociales del trabajo, así como la satisfacción personal. Se trata de un estudio transversal, en una muestra de 3084 trabajadores/as de salud de cinco municipios bahianos. Se utilizó un análisis de modelado de ecuaciones estructurales, estratificado por género. La insatisfacción con el trabajo fue la variable de exposición principal. Los TMC, variable de desenlace, se evaluaron mediante el Self-Reporting Questionaire (SRQ-20). Satisfacción personal (SAP), evaluada por cuestiones del World Health Organization Quality of Life (WHOQOL), y los aspectos psicosociales estresores $(A E)$ y protectores $(A P)$, medidos por el Job Content Questionnaire (JCQ) y por la escala EffortReward Imbalance (ERI), fueron tratadas como variables latentes. La insatisfacción con el trabajo se asoció positivamente a los TMC, entre hombres (0,160; IC95\%: 0,024; 0,295) y mujeres $(0,198$; IC95\%: 0,135; 0,260). Los AE se asociaron positivamente a la insatisfacción con el trabajo y a los $T M C$, mientras que los AP se asociaron negativamente a esos dos eventos, entre hombres y mujeres. La insatisfacción con el trabajo medió el efecto de los aspectos psicosociales y de la satisfacción personal en la ocurrencia de TMC. El modelo final presentó un buen ajuste en los grupos analizados. Elevada insatisfacción, presencia de aspectos psicosociales estresores, carencia de aspectos protectores e insatisfacción personal se asociaron a los TMC directa o indirectamente. Los resultados refuerzan la necesidad de acciones dirigidas a la protección de la salud mental en el trabajo en salud, con el rediseño de los aspectos nocivos identificados, así como el fortalecimiento de los aspectos asociados a una menor ocurrencia de TMC.

Estrés Laboral; Satisfacción en el Trabajo;

Trastornos Mentales
Recebido em 20/Ago/2020

Versão final reapresentada em 01/Nov/2020

Aprovado em 11/Dez/2020 